URLs

ORC2

http://ca.expasy.org/ uniprot/Q91628

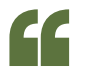

...mitotic

chromosome

remodelling

is ... required

for successful

nuclear

transfer.

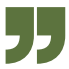

- Single-molecule analysis of the inter-origin spacing by molecular combing of DNA that was derived from fully differentiated erythrocyte nuclei, which were either first incubated in M-phase extract before S-phase induction (a) or directly incubated in S-phase extract (b). The DNA molecule is in red and nascent DNAs at replication origins are in green. Image courtesy of Jean-Marc Lemaitre, Institute of Human Genetics, Montpellier, France.

DNA REPLICATION

\title{
Turning back the genomic clock
}

Transplanting nuclei from differentiated cells to enucleated eggs - a process known as nuclear transfer - is a powerful, but also inefficient, technique to produce cloned animals. Reporting in Cell, Méchali, Lemaitre and colleagues have now uncovered an important determinant for adapting differentiated nuclei to the accelerated DNA replication that is seen in early Xenopus laevis development, which might impact on the success rate of nuclear-transfer experiments.

To understand how differentiated nuclei can be reprogrammed for the rapid DNA replication that is typical of early development, Lemaitre incubated differentiated erythrocyte nuclei either directly in extracts prepared from $X$. laevis eggs that were blocked in $\mathrm{S}$ phase or in a mitotic (or M phase) egg extract for 45 minutes prior to S-phase induction. Erythrocyte nuclei in the S-phase extract replicated less efficiently than undifferentiated sperm nuclei,

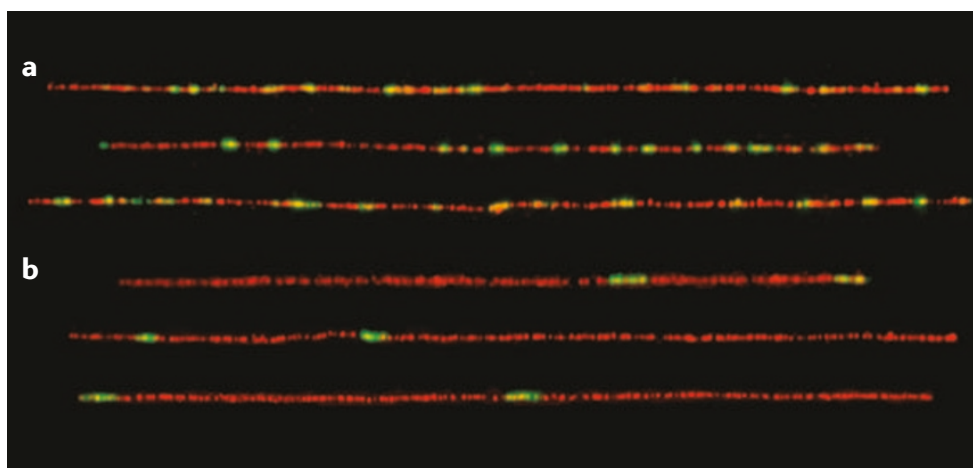

but erythrocyte nuclei that had been pre-treated replicated as rapidly and efficiently as undifferentiated sperm nuclei. So, passage through mitosis is important for the improved replication efficiency of differentiated nuclei.

The large number of replication origins in early $X$. laevis development allows a high rate of replication.

Replication origins are further apart in erythrocyte nuclei, but exposure to the M-phase extract led to a shortening of the spacing, resulting in a replicon size that is similar to that of sperm nuclei. This replicon remodelling could be explained by the shortening of the chromatin loops that was seen in erythrocyte nuclei that had been preincubated with mitotic extract prior to S-phase induction. This chromatin organization was similar to that of sperm nuclei entering $S$ phase.

Why is passage through mitosis important for reorganizing the nuclei for rapid replication? When erythrocyte

\section{RESEARCH HIGHLIGHTS ADVISORS}

GENEVIÈVE ALMOUZN

Institut Curie, Paris, France

JOAN S. BRUGGE

Harvard Medical School, Boston, MA, USA

IVAN DIKIC Goethe University Medical School,

Frankfurt, Germany

TOREN FINKEL

National Institutes of Health, Bethesda, MD, USA
PAMELA GANNON

Cell and Molecular Biology Online YOSEF GRUENBAUM The Hebrew University of Jerusalem, Israel

F. ULRICH HARTL

Max Planck Institute, Martinsried, Germany ELISA IZAURRALDE

Max Planck Institute, Tübingen, Germany
STEPHEN P. JACKSON

Wellcome Trust/Cancer Research UK Gurdon Institute, Cambridge, UK JENNIFER LIPPINCOTT-SCHWARTZ National Institutes of Health, Bethesda, MD, USA

NORBERT PERRIMON

Harvard Medical School, Boston, MA, USA nuclei were only briefly incubated in M-phase extract and then immediately induced into $S$ phase, the replication efficiency remained low. This implies that it is not the M-S-phase transition that is important, but rather the formation of mitotic chromosomes during $M$ phase. Indeed, erythrocyte nuclei do not condense in topoisomerase-II (TopoII)-depleted M-phase extracts, and when erythrocyte nuclei were incubated in an M-phase extract that contained a TopoII inhibitor, the rate of replication remained low. The recruitment of the replication protein ORC2 was reduced when TopoII activity was inhibited, so the TopoIIdependent mitotic remodelling is required for the efficient recruitment of replication proteins in $\mathrm{S}$ phase.

An equivalent TopoII-dependent chromatin reorganization occurred in vivo during mitosis in early $X$. laevis development. During S phase, the fusion of replicons leads to an increase in the mean DNA-loop size. These large loops are remodelled into small loops in mitosis, which allows an increased binding of replication proteins for a higher rate of DNA replication. This mitotic chromosome remodelling is an important element in the genetic reprogramming that is required for successful nuclear transfer.

Arianne Heinrichs

ORIGINAL RESEARCH PAPER Lemaitre, J.-M. et al. Mitotic remodeling of the replicon and chromosome structure. Cell 123, 787-801 (2005) 